\title{
Eficácia dos jogos virtuais como tratamento fisioterapêutico na doença de Parkinson: revisão integrativa
}

Efficacy of virtual games as a physical therapy treatment in Parkinson's disease: an integrative review Eficacia de los juegos virtuales como tratamiento de fisioterapia en la enfermedad de Parkinson: revisión integradora Rodrigo Silva PERFEITO ${ }^{1}$

Pâmella Dayanna César SANTOS ${ }^{2}$

${ }^{1}$ Mestre em Ciências da Atividade Física e Diretor do Instituto Fisart, 20260-130 Rio de Janeiro-RJ, Brasil

${ }^{2}$ Especialista em Traumato-Ortopedia pelo IAPS e Membro do Grupo de Estudos do Instituto Fisart, 20260-130 Rio de Janeiro-RJ, Brasil

\section{Resumo}

A doença de Parkinson (DP) se constitui por ser neurodegenerativa e progressiva, acometendo durante sua instalação os neurônios dopaminérgicos, localizados na substância negra e estriada do cérebro, causando redução dos níveis de dopamina. Em consequência, cede início aos comprometimentos motores e emocionais, tais como tremor, déficit da marcha, rigidez, bradicinesia, instabilidade corporal, depressão, alteração de humor e problemas no sono. O tratamento tradicional é medicamentoso pela ingestão de dopamina, contudo, a utilização da realidade virtual tem se destacado como um recurso complementar. Sendo assim, o objetivo da pesquisa foi verificar a eficácia dos jogos virtuais no tratamento da DP. Para isto, optamos por uma revisão integrativa de estudos publicados nos últimos 5 anos nas bases de dados: SciELO, PubMed, Lilacs e PEDro. Por considerações finais, foi possível perceber que os jogos virtuais são ferramentas importantes por propiciar ótimos resultados, ludicidade e assiduidade no tratamento.

Descritores: Doença de Parkinson; Jogos de Vídeo; Fisioterapia.

\section{Abstract}

Parkinson's disease (PD) is constituted by being neurodegenerative and progressive, which during its onset, affects dopaminergic neurons taking on the black and striated substance of the brain causing a reduction in dopamine levels, which in turn, gives in to compromised motor and emotional, such as: tremor, gait problems, stiffness, bradykinesia, body instability, depression, changes in mood and sleep. Traditional treatment is medicated by ingesting dopamine, however, the use of virtual reality has stood out as a complementary resource. Therefore, the objective of the research was to verify the effectiveness of virtual games in the treatment of PD. For this, we opted for an integrative review of studies published in the last 5 years in the databases: SciELO, PubMed, Lilacs and PEDro. For final considerations, it was possible to realize that virtual games are important tools for providing great results, playfulness and assiduity in the treatment.

Descriptors: Parkinson Disease; Video Games; Physiotherapy.

\section{Resumen}

La enfermedad de Parkinson (EP) está constituida por ser neurodegenerativa y progresiva, que durante su instalación afecta a las neuronas dopaminérgicas, transformándose en la sustancia negra y estriada del cerebro, provocando una reducción de los niveles de dopamina, que a su vez, cede a las deficiencias motoras. .y emocionales, tales como: temblores, problemas de marcha, rigidez, bradicinesia, inestabilidad corporal, depresión, cambios de humor y sueño. El tratamiento tradicional se medica mediante la ingestión de dopamina, sin embargo, el uso de la realidad virtual se ha destacado como recurso complementario. Por tanto, el objetivo de la investigación fue verificar la efectividad de los juegos virtuales en el tratamiento de la EP. Para ello, optamos por una revisión integradora de los estudios publicados en los últimos 5 años en las bases de datos: SciELO, PubMed, Lilacs y PEDro. Para consideraciones finales, se pudo constatar que los juegos virtuales son herramientas importantes para brindar grandes resultados, alegría y asiduidad en el tratamiento.

Descriptores: Enfermedad de Parkinson; Juegos de Video; Fisioterapia.

INTRODUÇÃO

O aumento populacional de pessoas idosas tem sido motivo de destaque no Brasil. A redução da taxa de mortalidade tem aumentado de forma considerável a expectativa de vida ${ }^{1}$. Estima-se que no Brasil, o número de indivíduos que possuem 60 anos ou mais, passou de 3 milhões em 1960 para 14 milhões em 2002, ou seja, um aumento de quase $500 \%$ em 42 anos $^{2}$. Para os próximos anos, é sabido que o número de idosos só tende a crescer ainda mais.

A segunda patologia neurodegenarativa com maior índice de prevalência em indivíduos

com mais de 60 anos de idade é a Doença de Parkinson (DP), onde em sua maioria, são sujeitos do sexo masculino. É uma patologia que afeta os neurônios dopaminérgicos localizados na substância negra e estriada do cérebro causando a redução dos níveis de dopamina, que são neurotransmissores ligados diretamente aos movimentos corporais ${ }^{3}$.

Pode ser classificada em 3 tipos: primário, que é o mais comum e com sintomas tradicionais; o secundário, que abrange diversos sintomas dificultando o diagnóstico; e o terciário, que é chamado de Parkinson Plus, provocando junto aos transtornos motores, comprometimentos no sistema nervoso central ${ }^{4}$.

Pensando nas evoluções dos sintomas, o tremor é o primeiro a ser percebido, sendo a dor o motivo de maior queixa. Também foi compreendido pelas escalas UPDRS-MDS - III e Schwab and England Activities of Daily Living Scale que o nível de escolaridade é um influenciador na aquisição da doença ${ }^{5}$.

Tais tremores vêm associados à diversas dificuldades motoras, tais como irregularidade da marcha, rigidez, bradicinesia e instabilidade corporal. Porém, caso sua gravidade seja maior, podem ocorrer outros sintomas não motores, como depressão, alteração de humor e problemas no sono ${ }^{6}$. Sua alta incidência pode ser considerada um problema de saúde pública e econômica, uma vez que se apresenta como uma das patologias com maior custo financeiro para tratamento ${ }^{1}$. 
Apesar da intervenção farmacológica com reposição de dopamina ser indispensável no tratamento, terapias fisioterapêuticas desempenham um papel imprescindível na recuperação do aprendizado motor, promovendo maior controle espacial, temporal e de execução de movimentos finos, contribuindo assim, para otimização das atividades funcionais do dia a dia ${ }^{7}$. Um desses tratamentos complementares é a cinesioterapia utilizando jogos virtuais.

Desde a década de 1950 os jogos virtuais vêm sendo desenvolvidos com bastante tecnologia e começaram a ser utilizados no âmbito da reabilitação motora, desempenhando importante auxílio no processo de tratamento de várias patologias que necessitam do treino do raciocínio rápido e movimentos controlados, como é o caso da $\mathrm{DP}^{8}$. Além de propiciar efeitos ao nível cognitivo e motor, geram diversificação da atividade, melhoram o condicionamento físico e otimizam a interação social. Desse modo, a reabilitação virtual contribui de forma ampla para a recuperação do portador da doença trabalhando o sistema cardíaco, musculoesquelético, sensorial, vestibular e neurológico ${ }^{9}$.

Pensando assim, o objetivo deste trabalho é o de verificar a eficácia dos jogos virtuais no tratamento da DP através de uma revisão integrativa.

Por relevância científica, acreditamos que pela DP apresentar um índice bastante considerável de população acometida, é de grande importância acadêmica a busca e o apontamento de novos e eficazes tratamentos que realmente foram testados cientificamente.

Como relevância social, é perceptível que a tecnologia virtual agrada indivíduos de diversas idades, podendo ser um poderoso aliado na área da saúde, em especial para a fisioterapia, otimizando o tempo de tratamento, além de torná-lo mais divertido e diversificado para o paciente.

MATERIAL E MÉTODO

- Tipo de pesquisa

Trata-se de Revisão Integrativa com estudos publicados nos últimos 5 anos. A revisão Integrativa tem o objetivo de reunir e condensar resultados de pesquisas experimentais e não experimentais sobre um delimitado tema ou questão, de maneira sistemática e ordenada, contribuindo para o aprofundamento do conhecimento do tema investigado.

\section{- Busca eletrônica nas bases de dados}

Foram utilizadas as bases mais usuais na área de saúde: SciELO, PubMed, Lilacs e
PEDro por meio dos descritores: Parkinson, Jogos virtuais e Fisioterapia em língua portuguesa e inglesa para confecção da tabela. Também foram pesquisados artigos excepcionalmente do ano de 2020 e 2021 e da língua portuguesa no Google Acadêmico para enriquecimento da introdução e da discussão.

- Critérios de inclusão e exclusão

Dentre os critérios de inclusão temos artigos publicados nos últimos 5 anos (20172021), sem ressalvas em relação à amostra e que possuíssem como tema jogos virtuais com o propósito de reabilitação para o DP. Foram excluídos estudos pagos, que não abordavam o tema de forma clara e que tinham como metodologia a revisão da literatura

- Extrações dos dados

Após selecionar os artigos da amostra final, foram extraídas algumas informações pertinentes de cada um deles para o uso da pesquisa, são elas: Referência da publicação; objetivo da pesquisa; caracterização da amostra e divisão ou não em grupos distintos de intervenção; forma de avaliação; características do protocolo aplicado, jogo utilizado, e resultados obtidos. Os resultados foram descritos através de tabelas e cronogramas. RESULTADOS

A busca nas plataformas resultou em um total de 75 artigos encontrados. Após aplicação dos critérios de inclusão e exclusão, foram selecionados um total de 7 artigos (Figura 1). Os resultados encontrados nos estudos selecionados estão apresentados na tabela 1 . Dentre as informações extraídas estão: autor/ano; amostra(n)/ idade/ grupos; método/instrumentos, duração/ intervenção e os resultados.

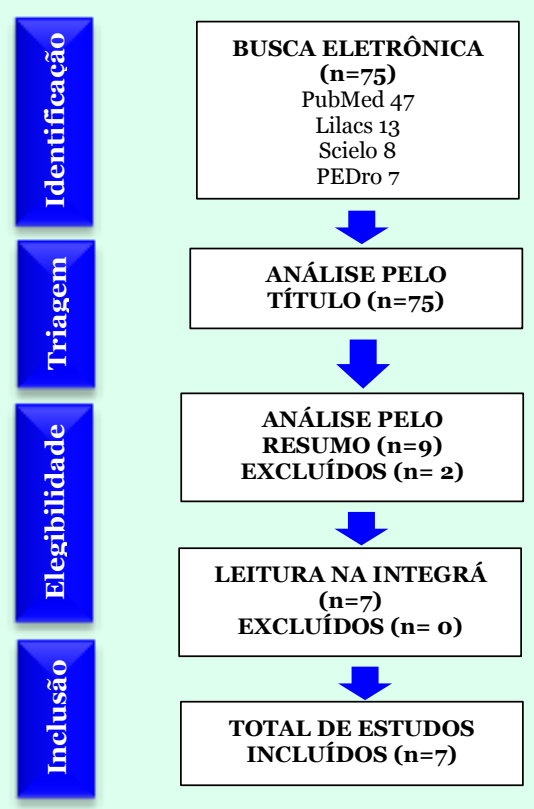

Figura 1: Fluxograma de busca nas plataformas. 
Tabela 1. Características dos artigos selecionados

\begin{tabular}{|c|c|c|c|c|}
\hline $\begin{array}{l}\text { Autor/ } \\
\text { Anos }\end{array}$ & $\begin{array}{l}\text { Amostra(n)/ } \\
\text { Idade/ } \\
\text { Grupos }\end{array}$ & $\begin{array}{l}\text { Método/ } \\
\text { Instrumentos }\end{array}$ & $\begin{array}{l}\text { Duração/ } \\
\text { Intervenção }\end{array}$ & Resultados \\
\hline $\begin{array}{l}\text { Souza et al. } \\
2018\end{array}$ & $\begin{array}{l}11 \text { indivíduos }(7 \\
\text { homens e } 4 \\
\text { mulheres) com } \\
\text { DP idiopática nos } \\
\text { estágios } 1 \text { a } 3 \text { da } \\
\text { doença e e idades } \\
\text { entre } 48 \text { a } 76 \\
\text { anos. }\end{array}$ & $\begin{array}{l}\text { MMS;UPDRS;MoCa; } \\
\text { Questionário (PDQ- } \\
\text { 39); Sistema Kinect } \\
\text { do X-Box } 360 .\end{array}$ & $\begin{array}{lrr}14 & \text { sessões } & \text { com } \\
\text { duração de } 1 \mathrm{~h} & 2 \mathrm{x} \\
\text { por } & \text { semana } \\
\text { durante } & 7 \\
\text { semanas. } & \end{array}$ & $\begin{array}{l}\text { Melhora } \\
\text { significativa nas } \\
\text { atividades de vida } \\
\text { diária; função de } \\
\text { evocação tardia } \\
\text { com leve aumento } \\
\text { no escore; Efeitos } \\
\text { positivos nos das das } \\
\text { domínios de vida } \\
\text { atividades de vida } \\
\text { diária (equilíbrio e } \\
\text { controle postural); } \\
\text { Leve melhora da } \\
\text { cognição. }\end{array}$ \\
\hline $\begin{array}{l}\text { Severiano } \\
\text { et al, } 2018\end{array}$ & $\begin{array}{l}16 \text { pacientes }(6 \\
\text { mulheres e 10 } \\
\text { homens) com DP } \\
\text { e idades entre } 18 \\
\text { e } 82 \text { anos. }\end{array}$ & $\begin{array}{l}\text { BBS;DHI;Medical } \\
\text { OutcomeStudy; } \\
\text { SF36;LRF;Nintendo } \\
\text { Wii, Wii-Remote e } \\
\text { Wii Balance Board. }\end{array}$ & $\begin{array}{l}20 \text { sessões de } 50 \\
\text { minutos cada, } 2 x \\
\text { por semana, } \\
\text { avaliação antes e } \\
\text { após o término } \\
\text { das sessões. }\end{array}$ & $\begin{array}{lr}\text { Melhora } & \text { na } \\
\text { habilidade } & \text { e } \\
\text { mobilidade } & \text { de } \\
\text { membros } & \\
\text { inferiores; } & \\
\text { Resultado } & \\
\text { significativo } & \text { na } \\
\text { saúde mental. } & \end{array}$ \\
\hline $\begin{array}{l}\text { Nuic et al. } \\
2018\end{array}$ & $\begin{array}{l}10 \text { paciente com } \\
\text { DP (5 homens e } 5 \\
\text { mulheres) com } \\
\text { idade média } \\
\text { entre } 64,2 \pm 61 \\
\text { anos. }\end{array}$ & $\begin{array}{l}\text { MMS;Plataforma de } \\
\text { forca (o,o9X1,8m, } \\
\text { AMT INc LG6-4-1); } \\
\text { Escala Likert;Piper } \\
\text { Fatigue } \\
\text { RevisedScale; } \\
\text { EPN-31;FOG-Q; } \\
\text { GABS; } \\
\text { Videogame } \\
\text { 'Toaprun'. }\end{array}$ & $\begin{array}{l}18 \text { sessões } \\
\text { durante } 6 \\
\text { semanas }\end{array}$ & 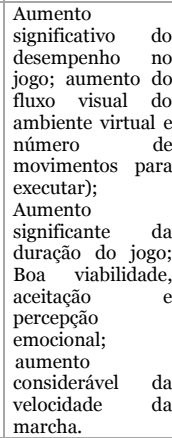 \\
\hline $\begin{array}{l}\text { Nogueira et } \\
\text { al. } 2017\end{array}$ & $\begin{array}{l}9 \text { pacientes com } \\
\text { DP (3 mulheres e } \\
6 \text { homens) com } \\
\text { idades entre } 56 \text { e } \\
77 \text { anos. }\end{array}$ & $\begin{array}{l}\text { YH; EEB;Nintendo } \\
\text { Wii fitplus, Testes de } \\
\text { normalidade de } \\
\text { Kolmogorov } \\
\text { Smirnov teste para } \\
\text { dados paramétricos } \\
\text { e Wilcoxon. }\end{array}$ & $\begin{array}{l}2 \text { sessões, 2x por } \\
\text { semana, com } \\
\text { duração de } 55 \\
\text { min, por } 10 \\
\text { semanas, } \\
\text { totalizando } 20 \\
\text { sessões }\end{array}$ & $\begin{array}{lr}\text { A adaptação aos } \\
\text { jogos } \\
\text { satisfatória; foi } \\
\text { Foi encontrado } \\
\text { aumento } \\
\text { significativo do } \\
\text { equilíbrio rorga } \\
\text { escala de Berg. } \\
\end{array}$ \\
\hline $\begin{array}{l}\text { Silva et al. } \\
2019\end{array}$ & $\begin{array}{lr}10 \text { pacientes } & 5 \\
\text { mulheres } & \text { e } \\
\text { homens) } & \text { com } \\
\text { idades entre } & 57 \text { e } \\
76 \text { anos. } & \end{array}$ & 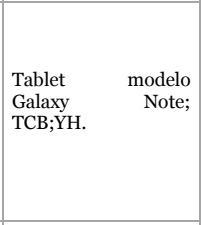 & $\begin{array}{l}16 \text { sessões } \\
\text { individuais, } 2 \mathrm{x} \\
\text { por semana com } \\
\text { duração de } 30 \\
\text { minutos cada, } \\
\text { com avaliação } \\
\text { inicial e } \\
\text { reavaliação pós } \\
\text { treinamento. }\end{array}$ & $\begin{array}{l}\text { Melhora das } \\
\text { habilidades } \\
\text { motoras manuais; } \\
\text { melhora ou } \\
\text { manutenção no } \\
\text { desempenho do } \\
\text { jogo. Otimização } \\
\text { da coordenação } \\
\text { motora fina e } \\
\text { destreza manual. }\end{array}$ \\
\hline $\begin{array}{c}\text { Santos et al, } \\
2019\end{array}$ & $\begin{array}{lrr}10 \text { pacientes } & 5 \\
\text { homens e } & 5 \\
\text { mulheres) re no } \\
\text { estágio 1 a } & 3 \text { da } \\
\text { doença r com } \\
\text { idades entre } & 55 \mathrm{e} \\
80 \text { anos. } & \end{array}$ & $\begin{array}{l} \\
\text { YH; Console X-Box } \\
\text { 36o TM com Kinect; } \\
\text { Aparelho de TV de } \\
\text { LED 42 polegadas; } \\
\text { Câmera digital Sony } \\
\text { Cyber-Shot DSC- } \\
\text { W80o 20.1 MP LCD } \\
2.7 .\end{array}$ & $\begin{array}{l}\text { Em cada nível, os } \\
\text { voluntários } \\
\text { jogaram duas } \\
\text { vezes, uma vez } \\
\text { com o us da } \\
\text { braçadeira de } \\
\text { o,5kg, e a a } \\
\text { segunda vez, sem } \\
\text { o uso da mesma. } \\
\text { A coleta foi } \\
\text { realizada ra no } \\
\text { mesmo dia, } \\
\text { durante ramente } \\
\text { aproximadamente } \\
\text { 5 minutos não } \\
\text { contínuos de jogo, } \\
\text { com rá cada } \\
\text { voluntário. }\end{array}$ & $\begin{array}{l}\text { Os resultados } \\
\text { mostram que não } \\
\text { houve diferença } \\
\text { significativa na } \\
\text { amplitude de } \\
\text { abdução de ombro } \\
\text { em relação ao uso } \\
\text { de carga durante } \\
\text { os jogos, aos níveis } \\
\text { dejogo, bem como } \\
\text { entre os membros } \\
\text { superiores direito } \\
\text { e esquerdo. }\end{array}$ \\
\hline $\begin{array}{l}\text { Costa et al. } \\
2018\end{array}$ & 5 pacientes & $\begin{array}{l}\text { MEEM; UPDRS; } \\
\text { YH; EEB;Timed Up } \\
\text { and Go, Wii Fit Plus } \\
\text { (Nintendo(C); SPSS; } \\
\text { Teste de Shapiro } \\
\text { Wilk; Teste de } \\
\text { Friedman. }\end{array}$ & $\begin{array}{l}4 r \text { intervenções, } \\
\text { em } 4 \quad \text { dias } \\
\text { diferentes, com } \\
\text { intervalo de sete } \\
\text { dias entre elas. }\end{array}$ & $\begin{array}{l}\text { Melhora apenas } \\
\text { na variável } \\
\text { posturográfica; } \\
\text { Houver uma } \\
\text { discreta melhora } \\
\text { no desempenho da } \\
\text { variável amplitude } \\
\text { ML no jogo. }\end{array}$ \\
\hline
\end{tabular}

\section{DISCUSSÃO}

De acordo com a Organização Mundial de Saúde (OMS) 1\% da população mundial acima de 65 anos são diagnosticados com a DP. Os sintomas geralmente se iniciam a partir dos 50 anos de idade ${ }^{10}$.

Na maioria dos estudos selecionados, as amostras foram compostas por pacientes com idades entre 49 a 75 anos, de ambos os sexos, porém, com predominância do sexo masculino.
O primeiro estudo da tabela objetivou analisar os efeitos da realidade virtual na cognição e qualidade de vida de pacientes com DP. Foi realizada uma avaliação inicial, imediatamente após a intervenção e 30 dias após. Foram selecionados de forma aleatória 4 jogos do Kinect Adventures; os jogos exigem rápido deslocamentos dos membros e controle postural. Sendo assim, após o termino das sessões a pesquisa concluiu que houve melhora no domínio de vida diária, porém, nos domínios avaliados quanto à cognição e a qualidade de vida não foram encontrados resultados relevantes ${ }^{11}$.

Já no estudo clínico piloto em idosos com DP onde o grupo de intervenção realizou 16 sessões de reabilitação pulmonar com jogos de realidade virtual, houve melhora na qualidade de vida. Os pacientes tinham comprometimento da expansibilidade da caixa torácica devido a fraqueza e rigidez muscular. Após as sessões, os resultados demonstraram que as pressões de inspiração e de expiração máximas melhoraram, o recrutamento de músculos acessórios diminuiu e houve um aumento da participação do diafragma, melhorando as condições respiratórias dos participantes, e consequentemente, como relatado, incremento da qualidade de vida ${ }^{12}$.

Pensando na cognição, um estudo experimental longitudinal avaliou os efeitos da gameterapia sobre a capacidade cognitiva de pacientes com DP. Apesar de metodologia não tão atraente, com apenas 6 participantes, os autores concluíram que a terapia através dos jogos virtuais provoca efeitos positivos na cognição $^{13}$.

A segunda pesquisa da tabela buscou verificar a eficácia dos exercícios de equilíbrio com a realidade virtual. Os pacientes realizaram avaliações otorrinolaringológica, anamnese e triagem do sistema vestibular juntamente com os demais testes aos quais foram realizados antes da reabilitação e após a $20^{\circ}$ sessão. Durante os encontros foi utilizado o Nintendo Wii-Remote e o Wii Balance Board. Quatro jogos de equilíbrio foram empregados (Soccer Heading, inclinação da mesa, caminhada na corda bamba e Ski Slalom) que envolviam estratégias de movimentos oculares, estímulos optocinéticos, coordenação motora, movimentos circulares pélvicos, equilíbrio estático e dinâmico, entre outros. Após análise dos resultados os escores finais do inventário de deficiência de tontura e equilíbrio de Berg apresentaram melhora após a reabilitação, o teste de sentar e levantar também apresentou resultado significativo, já o questionário SF-36 
demonstrou capacidade funcional significativa para os jogos Tightrope Walk e Ski Slalom ( $p<$ $0,05)$ e da saúde mental para o jogo Ski Slalom $(p<0,05)$. Todos os resultados garantem a eficácia dos jogos como opção terapêutica para essa população, ao qual foi relatada na rotina diária pelos próprios pacientes ${ }^{14}$.

Outro estudo que analisou os efeitos do Nitendo Wii no equilíbrio e marcha, testou a reabilitação dos participantes com 3 jogos (Penguin Slide, Plataforms e Balance Bubble), 2 vezes por semana, por 6 semanas. Os resultados demonstraram aumento da velocidade da marcha, aumento da distância entre os passos, maior agilidade e segurança ao andar e melhora do equilíbrio estático ${ }^{15}$.

O terceiro estudo da tabela buscou determinar a viabilidade e aceitabilidade da reabilitação através de um videogame para tratar distúrbios da marcha e equilíbrio, avaliando seus efeitos diante dos sinais motores apresentados na DP. Foi utilizado o Kinect com o jogo 'Toap Run' por 18 sessões, durante 6 semanas, onde o paciente precisava realizar deslocamentos com movimentos rápidos e de grandes amplitudes que exigiam a utilização de todo o corpo. Os resultados apresentaram altos escores de viabilidade, aceitabilidade e satisfação, além disso, o questionário de congelamento de marcha, escala de marcha e equilíbrio e pontuação axial apresentaram diminuição significativa de $41 \%$ e a escala de confiança do equilíbrio aumentou 35\%. Quanto a marcha, houve melhora do comprimento do passo e da velocidade, diminuindo o tempo de apoio duplo. Três meses após a última sessão não houveram mudanças significativas, apenas a persistência da diminuição da pontuação axial e aumento do comprimento e velocidade do passo ${ }^{16}$.

Em estudo semelhante houveram resultados não muito positivos para a melhora da marcha, demonstrando a necessidade de mais estudos. Avaliando a influência da realidade virtual sobre a satisfação com a terapia e a velocidade da marcha, foram realizadas 10 sessões com o console Nintendo Wii. Os resultados não demonstraram melhora na velocidade da marcha, porém, houve um aumento na satisfação de se reabilitar dos participantes ${ }^{17}$.

O quarto estudo da tabela avaliou 0 efeito da terapia por realidade virtual na postura de indivíduos com a DP. Foram utilizados 3 tipos de jogos do Nintendo Wii Fit Plus, são eles: Soccer Heading, o Penguin Slide e o Table Tilt, exigindo movimentos de tronco no sentido látero-lateral e manutenção do equilíbrio. Ao final da pesquisa houve melhoras significativas quanto as dimensões de provas estacionárias e na escala de equilíbrio de Berg. Dois dos paciente utilizavam dispositivo de auxílio para se manterem em ortostatismo na plataforma, porém, a mesma foi abandona por um dos pacientes decorrente da melhora da postura e equilíbrio ${ }^{13}$.

Em outro estudo também houve análise do equilíbrio postural, porém, em 6 pacientes hemiparéticos pós AVE com idade média de 58 anos utilizando como reabilitação jogos de realidade virtual por meio do Nintendo Wii durante 20 sessões. Apesar do baixo número amostral de apenas 6 participantes, que traz baixa confiabilidade, os resultados demonstraram melhora no equilíbrio estático e dinâmico por meio da escala de equilíbrio de Berg. A conclusão é que jogos de realidade virtual são eficazes para tratamento de pacientes que precisam de melhora postural ${ }^{18}$.

$O$ quinto estudo da tabela analisou as repercussões do treinamento com realidade virtual não imersiva nas habilidades motoras manuais de pacientes com DP. Foi utilizado um tablet com os aplicativos Dexteria®, Smash Hit e Dots que favorecem o feedback auditivo das tarefas. $O$ aplicativo Pinchit It do Dexteria® solicita dos pacientes a utilização da pinça bípode, já os Smash Hit e Dots utilizam a pinça trípode pelo treino com o uso da caneta do tablet. Ao término das sessões os pacientes apresentaram melhora ou manutenção de todas as habilidades trabalhadas nos jogos. Percebeu-se que houveram ganhos tanto nos membros dominantes como nos membros contra laterais ${ }^{19}$.

Em estudo que também avaliava as repercussões dos segmentos distais de membros superiores, foram utilizados jogos virtuais com auxílio de órtese de punho para tratamento da rigidez muscular em pacientes com DP. Perceberam que a tecnologia está cada vez ganhando mais importância no contexto social melhorando a qualidade de vida dos pacientes ${ }^{20}$.

O sexto estudo da tabela objetivou identificar a influência da adição de carga em diferentes níveis de dificuldades de jogos virtuais sobre a amplitude de movimento de abdução de ombro em pacientes com DP. O jogo "Vazamentos" foi selecionado com o intuito de estimular movimentos de membros superiores com abdução e flexão de ombro. O jogo foi dividido em nível básico (Marinheiro), nível intermediário (No Oceano) e nível avançado (Fonezi de Pesca). O nível Básico possui 3 etapas, cada uma de 45 segundos; o 
nível intermediário e avançado possuem 3 etapas, a primeira de 45 segundos e as demais de 60 segundos. De acordo com os resultados coletados a pesquisa concluiu que não houve diferença significativa na amplitude de abdução de ombro em relação ao uso ou não de carga durante o jogo e aos níveis de dificuldade dos jogos $^{21}$. Este fato pode ter ocorrido por falta de especificidade, já que o treino de força com uso de resistências serve para aumentar a força, e não a amplitude de movimento articular, no qual precisaríamos de exercícios de mobilidade ${ }^{2}$.

Um artigo que pode auxiliar na escolha do jogo correto de acordo com a intensidade pretendida, caso seu objetivo seja aumento de força, é o estudo experimental a seguir.

Analisando a intensidade de alguns jogos do Kinect Sports por variáveis hemodinâmicas e percepção do esforço por meio de 3 sessões em diversos jogos diferentes, foi entendido que alguns jogos apresentavam intensidade forte $(70$ a $89 \%$ da FCmáx), moderada (55 a 69\%) e baixa (35 a $54 \%$ ). Assim, com a escolha correta da intensidade do jogo combinado com o nível de aptidão do participante, é possível alcançar resultados mais relevantes ${ }^{22}$.

Assim como o segundo, o sétimo estudo da tabela teve como objetivo analisar 0 desempenho no equilíbrio de indivíduos com DP por meio de sessões com realidade virtual. Foram realizadas 4 intervenções em dias diferentes com intervalo de 7 dias entre elas. Todos os pacientes utilizaram 3 jogos diferentes do nível iniciante no console Nintendo Wii Fit Plus, são eles: Pinguim Slid, Table Tilt e Balance Bubble aos quais foram utilizados no segundo e terceiro dia de intervenção. Ao fim do estudo foi observado melhora no desempenho entre pré e pós intervenção principalmente no primeiro jogo (Pinguim Slid) ${ }^{23}$.

Buscando avaliar o equilíbrio em idosos e diminuir os riscos de queda após sessões com jogos do Nintendo Wii, uma revisão de literatura concluiu que esta intervenção, além de eficaz, possui boa aceitação por parte dos pacientes ${ }^{24}$. CONCLUSÃO

A maioria dos estudos utilizou como ferramenta 0 console Nintendo Wii com acessórios que adicionavam a realidade virtual para reabilitação dos portadores de DP, provavelmente pelo baixo custo e tecnologia mais simples comparado com os consoles e jogos de última geração lançados na atualidade. Apesar de uma pequena minoria ter encontrado efeitos mínimos, os resultados foram significativamente positivos quanto a melhora do equilíbrio, da postura, das habilidades de coordenação motora, agilidade corporal e melhor desempenho na marcha. Além disso, alguns estudos relataram maior aceitação ao processo de reabilitação e melhora da qualidade de vida.

Assim, após leitura extensa do assunto, é possível relatar que a reabilitação dos indivíduos com DP por meio de jogos virtuais é eficaz e pode ser utilizada como tratamento auxiliar aos medicamentos a base de dopamina. REFERÊNCIAS

1. Bovolenta T, Felício A. O doente de Parkinson no contexto das Políticas Públicas de Saúde no Brasil. Einstein. 2016;14(3):7-9.

2. Perfeito R. Método Pilates: Uma possível intervenção para a promoção da saúde no envelhecimento. 2. ed. - Rio de Janeiro: Kirios, 2021.

3. Ferreira J. et al . Gerontotecnologia para prevenção de quedas dos idosos com Parkinson. Rev Bras Enferm. 2019;72(suppl 2): 243-50.

4. Costa ANF, Piazza L, Gregório EC, Santos APM, Mesquita KGF, Rosa Neto F. Efeitos dos programas de exercícios físicos e fisioterapia em indivíduos com Parkinson. Fisioter Bras. 2016;17(1):79-83.

5. Lunardi M, De Oliveira A, Freitas, F. Evolução das manifestações clínicas de pacientes com doença de Parkinson. Arq Catarin Med. 2020; 49(4):41-54.

6. Galvão C, Pimenta F. Dança para Parkinson: uma revisão de literatura. Actas do $12^{\circ}$ Congresso Nacional de Psicologia da Saúde, Lisboa: ISPA. Instituto Universitário, 2018.

7. Silva LP, Duarte MPS, Souza CCB, Lins CCSA, Coriolano MGWS, Lins OG. Efeitos da prática mental associada à fisioterapia motora sobre a marcha e o risco de quedas na doença de Parkinson: estudo piloto. Fisioter Pesqui. 2019;26(2):112-19.

8. Nogueira PC, Silva AM, Kosour C, Reis LM. Efeito da terapia por realidade virtual no equilíbrio de indivíduos acometidos pela doença de Parkinson. Fisioter Bras, 2017;18(5):547-52.

9. Costa R, Ribeiro M. Utilização do Nintendo Wii: reabilitação virtual em pacientes com paralisia cerebral. Rev Psicol Saúde e Debate. 2018: 4(2):14-24.

10. Silva ME, Silva WM, Silva CAO, Silva JMM, Silva GCS, Silva ERB et al. Doença de Parkinson, exercício físico e qualidade de vida: uma revisão. BJD. 2020;6(9):71478-488.

11. Souza MFS, Bacha JMR, Silva KG, Freitas TB, Torriani-Pasin C, Pompeu JE. Effects of virtual rehabilitation on cognition and quality of life of patients with Parkinson's disease. Fisioter mov. 2018;31:e003112, 2018. 
12. Araújo LB, Freitas ACL, Campos JA, Guimarães JPK, Reis LM, Silva AM et al. Efeito da realidade virtual no recrutamento de fibras musculares e força muscular respiratória em pacientes com doença de Parkinson- estudo piloto. Fisioter Bras. 2020;21(4):343-54.

13. Nogueira MLC, Galvão ACDR, Almeida FEO, Calado TSA, Pimentel MM, Avelino MCF et al. Efeito da gameterapia sobre a capacidade cognitiva de indivíduos portadores de Doença de Parkinson. BJHR. 2020;3(5):12946-956.

14. Severiano MIR, Zeigelboim BS, Teive HAG, Santos GJB, Fonseca VR. Effect of virtual reality in Parkinson's disease: a prospective observational study. Arq Neuro-Psiquiatr. 2018; 76(2):78-84.

15. Belchior L, Almeida L. Influência do uso do Wii Fit sobre o equilíbrio estático e a marcha na doença de Parkinson. Acta Fisiatr. 2020;7(2): 107-12.

16. Nuic D, Vinti M, Karachi C, Foulon P, Hamme AV, Velter ML. The feasibility and positive effects of a customised videogame rehabilitation programme for freezing of gaitand falls in Parkinson's disease patients: a pilot study. J Neuroeng Rehabil, 2018;15(1):31.

17. Carvalho AC, Souza MC, Biazini PLA, Furuta DT, Moliterno AH, Uliam NRSouza M. et al. A influência da realidade virtual sobre a velocidade da marcha e avaliação da satisfação de indivíduos com doença de Parkinson. Colloq Vitae. 2020;12(3):1-9.

18. Macêdo JLC, Rodrigues LRS, Rodrigues APCP, Alencar FJ, Lopes LAMR. Influência da realidade virtual no equilíbrio de pacientes hemiparéticos pós-ave. BJHR. 2020;3(4): 10674-684.

19. Silva GLO, Ceron BM, Borba KM, Amaral DS, Marcelino JFQ, Coriolano MGWS et al. Repercussões do treinamento com realidade virtual não imersiva nas habilidades motoras manuais de pessoas com doença de Parkinson. Acta Fisiatr. 2019;26(1):43-8.

20. Oliveira LC, Lamounier EA, Andrade AO. Aplicação de Jogos Sério baseado em Realidade Virtual para Reabilitação de Pacientes com Doença de Parkinson por meio de uma Órtese de Punho. Anais do XXII Simpósio de Realidade Virtual e Aumentada (SVR). 2020,pp.306-12.

21. Santos LR, Sousa LR, Lopes CR, Dionísio J, Fenelon SB, Hallal CZ. Game terapia na Doença de Parkinson: influência da adição de carga e diferentes níveis de dificuldade sobre a amplitude de movimento de abdução de ombro. $\mathrm{R}$ bras Ci e Mov. 2017;25(4):32-8.

22. Silva ES, Cabral JSAS, Costa MC, Lopes LHB, Sousa TAA, Cardoso GA. Classificação da intensidade dos jogos Kinect Sports ${ }^{\circledR}$ em universitários através das variáveis hemodinâmicas e da percepção subjetiva de esforço. Motricidade. 2020;16(2):225-34.

23. Costa RCS, Silva Filho EM, Gomes CLA, Lima NMFV, Cacho RO, Cacho EWA et al. Treino de equilíbrio em pessoas com doença de Parkinson com uso de realidade virtual. Fisioter Bras, 2018;19(3):368-76.

24. Pimentel PT, Soares RR. O efeito da realidade virtual no equilíbrio de idosos. Saúde Dinâmica, 2020;2(4).

\section{CONFLITO DE INTERESSES}

Os autores declaram não haver conflitos de interesse

\section{AUTOR PARA CORRESPONDÊNCIA}

\author{
Rodrigo Silva Perfeito \\ Instituto Fisart \\ Rua Haddock Lobo 332, sala 105 - Tijuca \\ 20260130 Rio de Janeiro-RJ, Brasil \\ E-mail: rodrigosper@yahoo.com.br
}

Submetido em 20/02/2021 Aceito em 16/07/2021 